\title{
Examining patient safety education in pre-registration nursing curriculum: Qualitative study
}

\author{
Mansour Mansour \\ Acute Care Department, Faculty of Health, Social Care and Education, Anglia Ruskin University, Chelmsford, England. \\ Correspondence: Mansour Mansour, RN, PhD, PGCert Edua. Address: $4^{\text {th }}$ Floor William Harvey Buidling, Acute Care \\ Department, Faculty of Health, Social Care and Education, Anglia Ruskin University, Rivermead Campus, Chelmsford, \\ Essex, CM1 1SQ. England. Email: mansour.mansour@anglia.ac.uk
}

Received: June 12, 2013

Accepted: July 28, 2013

Online Published: August 9, 2013

DOI : 10.5430/jnep.v3n12p157

URL: http://dx.doi.org/10.5430/jnep.v3n12p157

\section{Abstract}

Background: Patient safety education has become an important tool to shape the future safety behaviour of health care professionals. The World Health Organisation has recently advocated eleven patient safety themes that need to be addressed in any health care educational curriculum. It is not known at this stage how far pre-registration adult nursing curriculum addresses these patient safety themes.

Aims: To examine the views of nursing students and faculty members on patient safety education in a pre-registration adult nursing curriculum in relation to the WHO' patient safety themes.

Method: Qualitative method, utilising 20 semi-structured interviews with pre-registration adult nursing students and faculty members (10 students and 10 faculty members). The data were analysed thematically.

Findings: Three main themes emerged from the participants' views: 'Not explicitly linked to patient safety', 'Mismatch between theory and practice' and 'The role of the mentor in practice'. The power imbalance between the students and staff in clinical placement appeared to have rendered many students reluctant to challenge unsafe practice.

Conclusion: Patient safety education needs to be more visibly taught in nursing education. The nursing curriculum' content, teaching and delivery methods may need to be reconsidered to better address the relevant WHO' patient safety themes, but also to accommodate the unique context of each nursing educational system.

\section{Key words}

Patient safety education, Pre-registration, Nursing curriculum, Student nurses, Faculty members

\section{I ntroduction}

Ensuring safe health care is one of the most challenging issues for health care organisations. Patient safety education for health care professionals can play an important role in raising health care professionals' awareness of modern patient safety thinking, and the delivery of safe patient care ${ }^{[1-4]}$.

Several studies have been conducted worldwide to assess the current status of patient safety in nursing education. A recent literature review on patient safety education in nursing suggested an increasing interest in researching this area ${ }^{[5]}$. For 
example, Attree et al. ${ }^{[6]}$ explored the patient safety aspects in English pre-registration nursing degree curriculum. Using qualitative data collection methods, the study found that there was a paucity of clear patient safety learning objectives in the nursing curriculum, with little emphasis on the system approach and its relation to modern awareness of patient safety. The UK Patient Safety Education Study Group investigated the formal and informal ways of patient safety learning among pre-registration students in four healthcare professions, including nursing ${ }^{[4]}$, and found that patient safety in the curriculum (including nursing curriculum) was largely implicit rather than explicit, with some apparent gaps in the curricular content on important patient safety topics, such as epidemiology of adverse events and root cause analysis. Milligan ${ }^{[7]}$ argued that to sustain any improvement in patient safety, nursing education has to undergo fundamental changes to the health care curricula, with emphasis on both the importance of system approach, and the role of Human Factors theory in creating a safety culture. These changes are likely to help the students to be more conscious of their own limitations, thus focusing on solutions within the complex system.

There has been an international drive to reform the patient safety curriculum for undergraduate health care professionals. In the US, Cronenwett et al. ${ }^{[8]}$ suggested a framework for incorporating Quality and Safety Education for Nurses (QSEN), which contains six core competencies: patient-centred care, teamwork and collaboration, evidence-based practice, quality improvement, safety and informatics, and related Knowledge, Skills and Attitude (KSA).This has prompted research into examining the integration of QSEN in nursing education. For example, Smith et al. ${ }^{[9]}$ carried out an online survey in the US to assess how far the six competencies of QSEN were addressed in Schools of Nursing. 572 US Bacculareate Schools of Nursing and 57 associate degree programs were included in the sample. The findings suggested that patient-centred care was most frequently addressed competency in the curriculum (95\%), followed by safety (89\%) and teamwork collaboration (82\%). Other studies adopted a more subjective approach to examine patient safety education in nursing curricula, by relying on the participants' perception of what constitutes patient safety elements in the curriculum. For example, a cross sectional survey was conducted on 193 public and private Japanese university-affiliated nursing schools in $2010^{[10]} .43 \%$ of the questionnaires were returned $(n=83)$, the results showed that $90 \%$ of nursing schools surveyed have integrated the topic of patient safety education into their curricula, such as theories and models of error, Human Factors, verifying patient identity, communication and criminal liability. However, 30\% reported devoting less than five hours to the topic, and less than $25 \%$ of schools covered failure mode and effects analysis and sharing adverse events with other institutions for learning. Due to the methodological limitations, the researchers acknowledged that the validity of their assessment has not been verified, as respondents were simply giving their perceptions of the nursing school's curriculum, which may not truly reflect the actual curricula content.

Chenot and Daniel ${ }^{[11]}$ carried out a survey to evaluate the current status of patient safety awareness among pre-licensure nursing students. The researchers developed and distributed the Health Care Professionals Patient Safety Assessment Curriculum Survey (HPPSACS) to 618 students in seven universities in the southeast of the US; $51 \%$ of questionnaires were returned $(n=318)$. The survey results showed sizeable variations in the students' responses to the 34-item scale survey and subscale on patient safety education, which may imply inconsistent approach to teaching patient safety in pre-licensure nursing students.

The logistical challenges associated with the designing of patient safety-friendly nursing curriculum were also highlighted in the literature. Milligan ${ }^{[7]}$ acknowledged that fitting new educational material to an already full curriculum is a challenging task, and suggested that any specific addition to the curriculum, such as Human Factors, should be linked, by examples, to the current content of the curriculum to facilitate the integration process. Other researchers argued that patient safety is still considered a relatively new concept and many educators may not be fully familiar with how to integrate patient safety learning into the existing curriculum ${ }^{[12]}$. Employing clinical nurses who are receiving continuous practice updates on patient safety issues and feeding that into clinical nursing placement, in conjunction with the focus on theoretical aspects of patient safety by faculty members, was suggested strategy to move beyond theoretical concepts of patient safety education and to increase the application of safety knowledge and competencies in nursing practice ${ }^{[13]}$. 
In 2011, The World Alliance for Patient Safety, on behalf of the World Health Organization (WHO), has launched the Patient Safety Curriculum Guide for Multi-Health Professionals ${ }^{[14]}$. The Guide contains eleven patient safety themes that need to be addressed in health care education curriculum (see Table 1). The aim of this initiative was to assist universities and schools in several health care fields, including nursing, to deliver consistent and structured patient safety education to improve patient care. Establishing evidence on the current status of patient safety education in nursing curriculum in relation to the proposed WHO Curriculum Guide would provide baseline information on the current status of patient safety education in nursing curriculum, and suggest areas of consideration in reforming the nursing education.

The aim of the study was to examine the student nurses and faculty members' views on the extent to which their pre-registration adult nursing curriculum (both theory and practice) addresses the WHO Patient Safety Education Guide for Multi-Professionals ${ }^{[14]}$.

Table 1. WHO Patient Safety Education Themes for Multi Health Professionals

\section{WHO Patient Safety Topics to be included in Health Care education}

Topic 1: What is patient safety?

Topic 2: Why applying human factors is important for patient safety?

Topic 3: Understanding systems and the effect of complexity on patient care

Topic 4: Being an effective team player

Topic 5: Learning from errors to prevent harm

Topic 6: Understanding and managing clinical risk

Topic 7: Using quality-improvement methods to improve care

Topic 8: Engaging with patients and carers

Topic 9: Infection prevention and control

Topic 10: Patient safety and invasive procedures

Topic 11: Improving medication safety

\section{Methods}

Descriptive, qualitative approach, utilising semi-structured interviews, was employed in this study. Data were collected in $2011^{[15]}$. The current study considered the students, the faculty members and their interpretations, perceptions, meanings and understandings as the study's key data. Underpinned by constructivist paradigm, the qualitative approach utilised in this study would allow the researcher to examine more closely the participants' experiences on patient safety education in pre-registration nursing training, and to capture how this phenomenon is socially constructed in their experiences and behaviours.

\subsection{Study setting}

The study was carried out in one university in the East of England, where pre-registration nursing education is delivered in three campuses, with two intakes of students recruited per academic year (March and September). Typically, the number of students in each intake ranged between 250-300 students across four branches (adult, child, mental health and learning disability), with the adult nursing branch representing more than $85 \%$ of the total number of students recruited. To qualify as registered nurses, the students have to pass twelve modules, eleven of which have practice elements, where the students attend placements in hospitals and skills sessions at the university. Within the university settings, teaching methods include lectures, group-work and practical skills sessions in the clinical skills laboratory. The students are assessed by 
variety of methods such as essays, exams, presentations and personal reflections. The practice elements of the modules are assessed by allocated mentors in the clinical placements. Clinical mentors are registered nurses who are employed by the hospitals or primary care settings to work clinically, but also have additional responsibilities of mentoring nursing students.

\subsection{Sample and data collection}

In-depth, semi-structured interviews were conducted with convenient sample of ten pre-registration adult nursing students (nine female and one male), and ten faculty members (seven female and three male) who were involved in pre-registration adult nursing teaching. The faculty members were all academic staff who were involved in teaching pre-registration nursing students. Six students were mature students, and four of them worked as Health Care Assistants before joining the nursing course. One faculty member was a PhD holder, eight held MSc degrees and one had a BSc, and their teaching experiences ranged from 4 to 24 years. The students were in their final year, and were likely to have covered most of the teaching delivered during their training. Recruitment emails were sent by a nursing administrator to all final year pre-registration adult nursing students and to the faculty members involved in the pre-registration adult nursing teaching. Those interested in participating in the study have emailed the researcher directly to arrange for an interview date and venue. During the interviews, all participants were asked about their views on how far the current nursing curriculum addressed the eleven WHO patient safety themes. Data collection ceased when data saturation was achieved. On average, each interview lasted for one hour, and was digitally recorded and transcribed verbatim by professional transcriptionist. Twenty interviews were eventually conducted with the participants.

\subsection{Ethical considerations}

The study was approved by the Research Ethics Committee in the university where this study took place. Participation in the study was voluntary. The researcher was new to the university at that time, and the invitation emails sent to the students and relevant documents made it clear that the researcher had not been involved in their teaching or assessment and would not do so in the remaining time before they graduate, which helped to assure the students that their views would not affect their teaching or assessment in anyway. All participants were informed that the data they provided would remain anonymous, known only to the researcher; and pseudonyms were assigned to all participants' names during data analysis. Before the interview, each participant signed a consent form.

\subsection{Data analysis}

Data analysis was constantly conducted alongside data collection. Using constant comparative method, data were coded, categorized and compared with other emerging data using NVivo software. Thematic analysis was conducted whereby codes were also compared with one another and similar codes were clustered into categories ${ }^{[16]}$. Thematic analysis seeks to describe patterns across qualitative data, and it is compatible with constructivist paradigm ${ }^{[17]}$. Through its theoretical freedom, thematic analysis provides a flexible and useful research tool, which can potentially provide a rich and detailed, yet complex account of data.

\subsection{Research rigour}

To ensure the research rigour, the researcher utilised several strategies which are guided by Guba and Lincoln ${ }^{[18]}$, criteria of credibility, transferability and dependability for trustworthiness in qualitative research. The faculty members and students' nurses who participated in this study were selected because they went through the journey of nursing training, albeit from different roles, and they were likely to have expert views on the quality of patient safety education in nursing training, hence contributing to the credibility of the study. Moreover, the constant comparison of the data that emerged from the participants' interviews ensured the credibility of the data collected and analysed ${ }^{[19]}$, and while the principal researcher (MM) carried out the initial data analysis and coding, the data were also coded separately by an independent external researcher, and the interpretation of the data analysis was largely consistent with both MM and the external researcher. Some participants' views, both students and faculty, were not consistent with the general trends which 
emerged from the data analysis, such views were often called deviant cases ${ }^{[20]}$. Those cases were pursued and analysed to enhance the credibility of data analysis.

The transferability of the data was ensured by providing a detailed description of the study context ${ }^{[18]}$. To achieve this aim, the research settings, the participants, and the context of relationships were outlined. Therefore, the reader can examine the study' setting and context, and conceptually generalise to their own, hence, extending the findings of this study beyond the current study context. The use of interview guide, the conduct of all interviews by the principle researcher, and the use of digital recorder to record all interviews, were all measures that the principle researcher has utilised to ensure the consistency in collecting and analysing the data, therefore improving the dependability of the data.

\section{Findings}

Three main themes emerged from the participants' views: Relevance to patient safety, Mismatch between theory and practice and the role of the mentor in practice.

\subsection{Relevance to patient safety}

The participants expressed a range of views in relation to how their nursing education was related to patient safety themes included in the eleven-themes WHO Patient Safety Curriculums Guide. These views ranged from not addressed at all, to being addressed but not linked to patient safety and to being implicitly linked to patient safety. For example, all students and almost all of the faculty members agreed that they had never come across the concept of Human Factors, which was one major topic of patient safety; consequently, they could not explain how it may relate to patient safety education:

No, I don't think I have [heard of it]. It [Human Factors] is not something that springs to mind. [Student interviewee 2]

When probing the students' understanding of what is meant by patient safety, many students expressed an ambiguity of what constitutes patient safety:

I think a lot of it [definition of patient safety] was left to our own initiative to work ... it wasn't always directly mentioned; just we know it’s all about patient safety. [Student interviewee 8]

Several faculty members also suggested that while their nursing teaching may have emphasized the importance of being competent in the nursing practice, it was often left to the students to conclude its relevance to patient safety. One faculty member gave an example of how teaching related to being an 'effective team player' was delivered in the course:

I don't think that it [teaching related to being effective team player] is necessarily related to issues around patient safety, it's more identifying a critical incident that is actually happened and what you can learn from that incident, so I don't think necessarily that patient safety is picked up as a theme within that. [Faculty member interviewee 6]

Some patient safety issues which were taught in the nursing program seemed to have been given considerable attention in the curriculum, but often with no explicit link to patient safety. For example, the importance of reporting drug errors seemed to have been conveyed to nursing students as a task-oriented nursing responsibility, without necessarily understanding the underlying patient safety principles which drive this attitude:

If there was a problem with something like a drug error, as students, they would often think of it in terms of managing it as a bureaucratic issue, so they would want to fill out the paperwork to record the incident rather than necessarily think of it in terms of an incident which needs to be managed from a safety perspective. [Faculty member interviewee7] 


\subsection{Mismatch between theory and practice}

Many students in this study suggested that there were disparities between what they were taught in the university and what they practiced in clinical settings in relation to patient safety agenda. For example, the seemingly low-fidelity clinical simulation skills that the students received in their laboratory training in the university appeared to represent clear challenge for them when they tried to apply these skills in clinical placements, as such training was not always perceived to simulate the reality in clinical practice:

When you're actually in practice and you've got 13 IVs [Intravenous drugs], bloods and everything else, there is a lot more going on than what you realise from theory. They [university skills session] don't take into consideration that you've got a million things going on, a million interruptions [in placement]. [Student interviewee 8]

Furthermore, many students suggested that the clinical skills which they were taught at the university were presented to them in a simplistic manner, which did not take into consideration the contextual culture of clinical placements. For this reason, when the students attempted to practice the skills being taught in the university, they felt unprepared to manage what they perceived as challenging situations, particularly if they wanted to highlight unsafe practice in the placement. This situation seemed to have rendered many students powerless, with no choice but to adapt to the institutionallymodified practice standards, although these may not always be perceived by them to constitute the safest practice:

Because in textbook it is like, something happens, you don't agree with it, you challenge it and it gets sorted. But in real life practice it's not like that, because something bad will happen, you challenge it, there's conflict and then it makes your life more difficult because people don’t want to work with you. [Student interviewee 4]

Several faculty members implied that elements of theory-practice gap do exist in the patient safety education in the nursing training, but they tent to blame the inconsistent application of teaching in clinical practice. One faculty member gave an example of how risk assessment tools were used in different hospitals:

Lots of hospitals have their own documentation, and the actual risk assessment tools they use are likely to be local and therefore need to be taught in practice ... different hospitals use different tools, so you can't say this is a document you use and this is how you use it, you can only give an example of it. [Faculty member interviewee 1]

So both the students and faculty members seemed to have consistent views on the elements of mismatch between what the students were being taught in university, and what they were experiencing in their placement. The clinical mentors appeared to play a vital role in articulating the students' experiences when they face such dilemma in the clinical setting. The next section shed more light on the mentor role in this context.

\subsection{The role of the mentor in practice}

Several students suggested that their clinical mentors were seen by students as inspiring role models for practicing patient safety:

Like with controlled drugs, my mentor would make me stay and watch the process ... she always asked me to work out how much to give, with morphine and things like that, she'd ask me to do the working out just to make me think, instead of just sitting there watching to get me involved at work. [Student interviewee 9]

On the other hand, some students expressed their scepticism concerning the patient safety practices of their mentors, yet they were not always able to challenge the latter because clinical mentors are responsible for the students' clinical assessment (i.e. signing off their practice book). Many students suggested that to challenge the mentors on any safety issue would negatively impact on their clinical assessment grades. According to several students, the ward culture, previous 
health care experience and how senior or junior the student nurses in their course were all contributed to their perceived power imbalance with their clinical mentors:

They [clinical mentors] can't sign you off for challenging them, but I think when you're a junior nursing student that's a fear that you probably have but you don't want to challenge them or get off on a bad foot with them for fear of them not signing you off for that placement. [Student interviewee 4]

The faculty members, in general, do accept that there was a perceived power imbalance between the students and their clinical mentors, although some of them suggested that lack of students' knowledge might have contributed to the students' perceptions on this matter.

They [student' nurses] want to get the book signed off, so they don't want to challenge anybody. Sometimes the student is not sure of what to challenge, they themselves don't have the in-depth knowledge to say: this is what the literature says, or this is what we have been taught in the university. [Faculty member interviewee 6]

Several faculty members suggested other factors, such as age and previous work experience in health care settings, could play an importance role in the students' attitude toward challenging unsafe practice:

It's easier for somebody who is older, more confident and more comfortable with their role to challenge somebody’s judgment than for somebody who's relatively junior. [Faculty member interviewee 4]

So the student's ability and skills to challenge the perceived unsafe practice, including those related to the clinical mentors practice, would seem to relay mostly on the working culture of the clinical placement, and the personal and demographical characteristics of the student nurses, and probably less on the core nursing values that the students are expected to learn from the university level.

\section{Discussion}

The findings from this study suggest that most of the patient safety topics advocated by the WHO Patient Safety Curriculum Guide ${ }^{[14]}$ were either not addressed, or at the best taught to students but not necessarily linked to patient safety. This seems to be embedded not only in the written nursing curriculum, but also in the faculty teaching approach. This is supported by the findings of a previous study which suggested that patient safety was not explicit in the formal, written curriculum ${ }^{[6]}$. Other studies adopted less structured approach in investigating patient safety education ${ }^{[13]}$, or have a markedly different focus for the patient safety investigated and used other research methodologies ${ }^{[11]}$, therefore, their results may not be comparable with those of the current study, yet they have all demonstrated that patient safety education was not explicitly addressed in the nursing curriculum. In the context of the US, studies on the quality and safety contents in pre-licensure nursing education using the QSEN framework confirmed the current findings, and reported that all patient safety competencies were embedded, in various magnitudes, in undergraduate nursing education, with patient-centred care being the most visibly addressed in nursing education ${ }^{[9]}$. However, it was less clear whether these topics were linked to patient safety, or whether they were addressed as general, stand-alone issues in the curriculum.

The results from this study endorse previous findings on the theory-practice gap in nursing education, whereby students and newly qualified nurses often emerged from their courses with consistent set of 'high' professional nursing values, which were often thwarted in practice ${ }^{[21]}$. The current study also suggests that patient safety education continues to represent a challenge for bridging the theory-practice gap. For example, several students in this study advocated that the low-fidelity clinical simulation to which they were exposed to at the university level did not always match the reality in clinical practice, and they were often left struggling to cope with work complexities that were largely absent in their theoretical learning. Although low-fidelity clinical simulation in nursing may facilitate experiential learning and 
behaviour modifications for students nurses ${ }^{[22]}$, there is also strong evidence suggesting that high-fidelity simulators can help undergraduate nursing students to value the opportunity to practice nursing activities in a safe environment prior to clinical placement, and give them the opportunity to develop clinical competency prior to real-life practice ${ }^{[23,24]}$. More research is needed in this regard to provide more evidence on the benefits of using low, intermediate and high-fidelity simulation in patient safety education.

The participants' views in this study highlighted how the clinical mentor' role was socially constructed in the participants' narrative, and what was unique about their experience is how they contextualised the influence of communication, power dynamics and the socialisation of students with the nursing staff in the clinical placement, but also how the clinical mentors can have a crucial role in setting the parameters for the 'norms' of safe practice. Most of the participants seemed to acknowledge such phenomena, whereby the clinical mentors can have a paradoxical influence on the students' patient safety experience, by enforcing patient safety behaviours in one context, but not allowing them to develop important parameter of patient safety behaviour in another, such as speaking up against their perceived unsafe practice. Clinical nursing mentors' attitudes, experience and knowledge were said to influence nursing students' experience ${ }^{[25]}$, and the student nurses, in particular, are unlikely to have constructed such image of the mentor role in isolation, rather against the backdrop of their shared understanding, practice and experience of working with clinical mentors, suggesting that clinical nurse mentors' attitudes, teaching style and feedback may have significant impacts on shaping the future patient safety behaviours of their students. A qualitative study in Turkey investigated the pre-registration nursing students' views of their clinical mentors ${ }^{[26]}$. The findings suggested that feedback from mentors was important and that mentors should be motivational, not critical, when providing feedback to the students. Providing motivational feedback helps to improve the communication between mentors and students, but also increases the student' self-confidence to challenge ${ }^{[27]}$. In addition, nursing students were found to give more importance to the assessment and evaluation than do mentors ${ }^{[28]}$. In the UK context, the students' mentors are responsible for passing or failing the students' practice ${ }^{[29]}$, and this appeared to have deterred many students from voicing their concerns, at least until they got their practice book signed off (i.e. passing their clinical practice assessment). Speaking up against unsafe practice is a fundamental principle in safeguarding patient safety ${ }^{[30,31]}$. The current findings suggest that this message may have not been conveyed to the students appropriately in their nursing training, but also, the WHO Patient Safety Curriculum Guide ${ }^{[14]}$ may have not provided the necessary tool to address this issue adequately. Evidence from the literature suggests similar trends among nursing students ${ }^{[32]}$. For example, a qualitative study which involved thirteen pre-registration nursing students in UK ${ }^{[33]}$ found that in situations that called for nursing students to 'speak up', students are often silenced in clinical practice, but to overcome this they negotiate situations in an attempt to be heard. The fear of failing clinical placement was also an influential factor in the students' decision to challenge their mentors, or indeed any other member of staff ${ }^{[34]}$. Therefore, the clinical mentors may need to be more receptive to the students' concerns on patient safety, by allowing the students' skills to challenge unsafe practice to emerge in a constructive manner. Acting as a positive and inspiring role model will help to nurture such students' behaviour, but may also dictate the need for revisiting the mentorship curriculum to facilitate more effective mentor engagement with the students, and to allow greater emphasis on the duty of student nurses to speak up and report any patient safety concerns. In addition, the recently published Francis Report ${ }^{[35]}$ on failing care in Mid Staffordshire in England recommended that nursing schools should review its training programmes to promote open culture in the clinical work environment, and to embed the staff' courage to challenge unsafe practice as one important pillar for promoting openness in the health care system.

Achieving safe patient care should underlie all clinical nursing education ${ }^{[36]}$. When a student's behaviours pose a threat to patient safety in clinical placement, the nurse educator and mentor may subject the student to a failing grade, which may in turn influence future students' understanding of the concept of safe patient care. More research is needed to further explore how clinical mentors can transform the process of student' assessment in clinical placement into a learning opportunity for improving patient safety. Interestingly, many of the faculty members in the current study seemed to be aware of the power imbalance in the student-mentor relationships, yet they appeared powerless to challenge this situation. The students' ability to challenge unsafe practice and speak up their concerns does not seem to be highlighted sufficiently in the eleven 
themes of the WHO Patient Safety Curriculum Guide ${ }^{[14]}$. Such skill is a fundamental part of the non-technical skills required for safe clinical practice, and is important elements of patient safety education ${ }^{[37]}$. Even other themes, such as being effective team player, seemed to have little focus on how to empower nurses, as well as other health care professionals, to express their concerns, taking into considerations the working culture and the unique educational system in each setting.

\section{Conclusion}

The recently launched WHO Patient Safety Curriculum Guide for Multi-Professionals ${ }^{[14]}$ is an important step in integrating patient safety education in health care curricula, including nursing curriculum. Findings from this study suggest that many of the eleven patient safety topics published in the WHO Patient Safety Curriculum Guide were not explicitly linked to patient safety in academic settings. The mismatch between theoretical teaching at the university level and the application of knowledge in clinical placement meant that many nursing students were struggling to consolidate their clinical skills in practice. The study also highlighted the pivotal role of clinical mentors in enforcing students' patient safety experience and being an inspiring role model. However, both students and faculty members agreed that there is visible power imbalance in the student-mentor relationship, which hinders the ability of the former to challenge unsafe practice. The current version of the WHO Patient Safety Curriculum Guide does not seem to have addressed this issue adequately. The Guide may need reconsideration to include issues on empowering nursing students, taking into consideration the working culture and dynamic of relationships with heath care professionals in clinical placement settings.

\section{Study limitations}

The study was carried out in one UK academic institution, and although the findings may be transferable beyond this context, caution needs to be exercised in doing so. The researcher who conducted this study was employed by the academic institution where this study took place, and this may have inhibited some participants from expressing their views. However, the views expressed in the findings suggest that this concern was minimised, but might have not been fully neutralised. This study focused on the student nurses and faculty members' views only. The inclusion of the clinical mentors' in the research sample might have enriched the data analysis, and provided fresh perspectives of the students' experiences of patient safety education in practice. Future research may consider quantitative approach, such as factor analysis, to construct validate the themes obtained from the participants in this study by using large sample size, and to outline more visibly the theory-practice gap from clinical mentors, faculty members and students perspectives.

\section{I mplications for future practice}

Clinical mentors, students and faculty members may all reflect on these findings to improve the quality of clinical education, including patient safety education for nursing students. There may be a need to reconsider the current nursing mentorship education to help delivering a more tailored patient safety related skills for student nurses in clinical placement.

\section{Acknowledgements}

The authors would like to thank the student nurses and faculty members who participated in this study. Also, thanks go to Ernest Ampadu, PhD, for his assistance in data analysis and finalising the data codes, and to Professor Sharon Andrew from Faculty of Health and Social Care and Education at Anglia Ruskin University for her advice and support in critically reading the manuscript. Also, the author would like to thank Dr Agnes Leotsakos and Dr Hao Zheng, from the WHO Patient Safety Program, for their advice and assistance in utilising the WHO Patient Safety Curriculum Guide in this study. 


\section{References}

[1] National Steering Committee on Patient Safety. Building a Safer System: A National Integrated Strategy for Improving Patient Safety in Canadian Healthcare. Available from:http://rcpsc.medical.org/publications/building_a_safer_system_e.pdf (accessed 9th August 2011).

[2] Greiner, A. Knebel, E. Health Professions Education: A Bridge to Quality. Institute of Medicine Committee on the Health Professions Education Summit. Washington DC. 2003.

[3] House of Commons Health Committee. Patient Safety: 6th Report of 9 sessions 2008-09. London: Stationery Office; 2009.

[4] Pearson et al. Patient safety in health care professional educational curricula: examining the learning experience 2009. Available from: http://www.ncl.ac.uk/medev/assets/documents/Patientsafetyfullreport.pdf. (accessed 18th September 2012).

[5] Mansour, M. Current Assessment of Patient Safety Education. British Journal of Nursing. 2012; 21(9): 536-43. PMid:22585267

[6] Attree, M. Cooke, H. Wakefield, A. Patient safety in an English pre-registration nursing curriculum. Nurse Education in Practice 2008; 8(4): 239-248. PMid:17945535 http://dx.doi.org/10.1016/j.nepr.2007.09.003

[7] Milligan, F.J. Establishing a culture of patient safety - the role of education. Nursing Education Today. 2008; 27(2):95-102. PMid:16713030 http://dx.doi.org/10.1016/j.nedt.2006.03.003

[8] Cronenwett, L. Sherwood. G, Barnsteiner. J, Disch. J, Johnson. J, Mitchell. P, Sullivan. DT, Warren. J. Quality and safety education for nurses. Nursing Outlook. 2007; 55 (3): 122-31. PMid:17524799 http://dx.doi.org/10.1016/j.outlook.2007.02.006

[9] Smith, L. Cronenwett, L. Sherwood, G. Current assessments of quality and safety education in nursing. Nursing Outlook. 2007; 55(3): 132-137. PMid:17524800 http://dx.doi.org/10.1016/j.outlook.2007.02.005

[10] Maeda S, Kamishiraki E, Starkey J, Ehara K. Patient safety education at Japanese nursing schools: results of a nationwide survey. BMC Res Notes. 2011; 4: 1-33. PMid:22005273 PMCid:PMC3215394 http://dx.doi.org/10.1186/1756-0500-4-416

[11] Chenot, T. Daniel, L. Frameworks for Patient Safety in the Nursing Curriculum. Journal of Nursing Education 2010; 49(10): 559-568. PMid:20669876 http://dx.doi.org/10.3928/01484834-20100730-02

[12] Walton M, Woodward H, Van Staalduinen S, Lemer C, Greaves F, Noble D, Ellis B, et al. The WHO patient safety curriculum guide for medical schools. Quality and Saftey in Health Care. 2010; 19: 542-546. PMid:21127112 http://dx.doi.org/10.1136/qshc.2009.036970

[13] Vaismoradi, M. Salsali, M. Marck, P. Patient safety: nursing students' perspectives and the role of nursing education to provide safe care. International Nursing Review. 2011; 58(4): 434-442. PMid:22092321 http://dx.doi.org/10.1111/j.1466-7657.2011.00882.x

[14] World Alliance for Patient Safety. Patient Safety Curriculum Guide: Multi-professional Edition. Available from: http://whqlibdoc.who.int/publications/2011/9789241501958_eng.pdf. (accessed 12th June 2013).

[15] Silverman, D. Doing Qualitative Research: a Practical Handbook. London: Sage; 2005.

[16] Ritchie, J. Spencer, L. Qualitative data analysis for applied policy research. In: Bryman, A. Burgess, G. Analyzing qualitative data, ed. London: Routledge; 1994; 173-94.

[17] Braun V. Clarke V. Using thematic analysis in psychology. Qualitative Research in Psychology. 2006; 3(2): 77-101. http://dx.doi.org/10.1191/1478088706qp063oa

[18] Guba, E. and Lincoln, Y.Competing Paradigms in Qualitative Research. Handbook of Qualitative Research. Denzin, N. and Lincoln, Y. Thousand Oaks: CA Sage Publications, Inc; 1994; 105-177.

[19] Guba, E. G., Lincoln, Y. S. Paradigmatic controversies, contradictions, and emerging influences. In N. K. Denzin Y. S. Lincoln,eds. The Sage Handbook of Qualitative Research (3rd ed.), Thousand Oaks: CA: Sage; 2005; 191-215.

[20] Silverman, D. Interpreting Qualitative Data: Methods for Analysing Talks, Text and Interaction. London: Sage Publications; 2005.

[21] Gallagher, P. How the metaphor of a gap between theory and practice has influenced nursing education. Nurse Education Today 2004; 24(4):263-268. PMid:15110435 http://dx.doi.org/10.1016/j.nedt.2004.01.006

[22] Wilson, M. Shepherd, I. Kelly, C. Pitzner, J. Assessment of a low fidelity human patient simulator for the acquisition of nursing skills. Nurse Education Today. 2005; 25(1): 56-67. PMid:15607248 http://dx.doi.org/10.1016/j.nedt.2004.10.004

[23] Reilly, A. Spratt, C. The perceptions of undergraduate student nurses of high-fidelity simulation-based learning: A case report from the University of Tasmania. Nurse Education Today. 2007; 27(6): 542-550. PMid:17069935 http://dx.doi.org/10.1016/j.nedt.2006.08.015

[24] Weaver, A. High-Fidelity Patient Simulation in Nursing Education: An Integrative Review. Nursing Education Perspectives. 2011; 32(1): 37-40. PMid:21473481 http://dx.doi.org/10.5480/1536-5026-32.1.37

[25] Nahas, V. Yam, B. Hong Kong nursing students’ perceptions of effective clinical teachers. Journal of Nursing Education. 2001; 40, 233-237. PMid:11355764 
[26] Elcigil, A., Sari, H.Y. Students' opinions about and expectations of effective nursing clinical mentors. Journal of Nursing Education. 2008; 47(3): 118-12. PMid:18380265 http://dx.doi.org/10.3928/01484834-20080301-07

[27] Löfmark, A., Wikblad, K. Facilitating and obstructing factors for development of learning in clinical practice: A student perspective. Journal of Advanced Nursing. 2001; 34: 43-50. PMid:11430605 http://dx.doi.org/10.1046/j.1365-2648.2001.3411739.x

[28] Lee, W. Cholowski, K., Williams, A. Nursing students' and clinical educators' perceptions of characteristics of effective clinical educators in an Australian university school of nursing. Journal of Advanced Nursing. 2002; 39: 412-420. PMid:12175350 http://dx.doi.org/10.1046/j.1365-2648.2002.02306.x

[29] Nursing and Midwifery Council The Code: Standards of Conduct, Performance and Ethics for Nurses and Midwives. Available from: http://www.nmc-uk.org/Publications/Standards/The-code/Introduction/.(accessed 18th September 2012).

[30] Entwistle. VA, McCaughan. D, Watt. IS, Birks. Y, Hall. J, Peat. M, Williams. B, Wright. J; Patient Involvement in Patient Safety Group. Speaking up about safety concerns: multi-setting qualitative study of patients' views and experiences. Quality and Safety in Health Care. 2010; 19(6): 1-7.

[31] Lyndon. A, Sexton. B, Simpson, K, Rosenstein. A, Lee. K, Wachter. R, Predictors of likelihood of speaking up about safety concerns in labour and delivery. British Medical Journal Quality and Safety. 2011; 21(9):791-9.

[32] Levett-Jones, T. Lathlean, J. Don’t rock the boat: Nursing students' experiences of conformity and compliance Nurse Education Today. 2009; 29(3): 342-349.

[33] Bradbury-Jones, C Sambrook, S. Irvine, F. Empowerment and being valued: A phenomenological study of nursing students' experiences of clinical practice. Nurse Education Today. 2011; 31 (4): 368-372. PMid:20696505 http://dx.doi.org/10.1016/j.nedt.2010.07.008

[34] Bellefontaine, N. Exploring whether student nurses report poor practice they have witnessed on placements. Nursing Times. 2009; 105(35): 28-31. PMid:19791676

[35] Francis, R. Report of the Mid Staffordshire NHS Foundation Trust Public Inquiry: Volume II. 2013. Available from: http://www.midstaffspublicinquiry.com/sites/default/files/report/Volume\%202.pdf(accessed 26th April 2013).

[36] Tanicala, M. Scheffer, B. Roberts, M. Defining pass/fail nursing student clinical behaviours phase I: moving toward a culture of safety. Nursing Education Perspectives. 2011; 32(3): 155-61. PMid:21834376 http://dx.doi.org/10.5480/1536-5026-32.3.155

[37] Flin, R. Patey, R. Improving patient safety through training in non-technical skills. British Medical Journal. 2009; $339:$ b3595 PMid:19776108 http://dx.doi.org/10.1136/bmj.b3595 